\title{
Remote magnetic navigation for ventricular ablation: did the machine win this round?
}

\author{
J. David Burkhardt ${ }^{1}$
}

Received: 22 May 2016/Accepted: 2 October 2016/Published online: 8 October 2016

(C) Springer Science+Business Media New York 2016

Remote magnetic navigation has been available as a tool for mapping and ablation for well over a decade. In that period of time, it has shown to be useful in nearly every type of ablation procedure from the most simple to the most complex [1-3]. Some operators prefer the system for atrial fibrillation ablation, others for ventricular tachycardia ablation, and some use it for any ablation. Despite numerous publications showing reductions in fluoroscopic exposure and the obvious benefits of not wearing lead and standing tableside for hours, remote magnetic navigation has not become the standard of care in the world [4]. Most publications appear to show similar success rates, and many show favorable complication rates [5-7]. So what is the limitation to universal acceptance? In addition, many centers have purchased the system, yet it sits unused. Why would a physician give up a more comfortable procedure to perform an ablation wearing lead and being exposed to more radiation?

One of the major factors preventing adoption is cost. Not only is the system itself expensive but it also requires shielding, specific X-ray systems, support specifications, a significant space requirement, and cannot be close to an MRI system. Outfitting an existing EP laboratory with a system would likely require significant construction costs. In general, it is much easier to plan for a system in new construction.

Once the system is installed, the operator must commit to overcoming the learning curve. Navigation with the

J. David Burkhardt

jdavidburkhardt@hotmail.com

1 Texas Cardiac Arrhythmia Institute, 3000 N. IH 35, Suite 700, Austin, TX 78705, USA system is quite different than manual navigation. Looping the catheter allows access to areas that cannot be reached directly. There is no fixed curve, which allows great versatility but is not always intuitive. Procedures will take longer in the beginning, and learning the advanced techniques takes significant time and patience. In the current world, where we are pushed toward efficiency and increasing volume and throughput, this time is certainly a sacrifice. It is understandable why some would choose not to continue using a system that slows them down under such pressure.

Perhaps the most challenging limitation is technological lag. Magnetic navigation systems require partnerships with catheter manufacturing companies and mapping companies to fully enjoy use of the system. These companies have their own financial goals, and since magnetic navigation is only a small portion of catheter sales, the incentive to advance the technology is not present. Since the release of the open-irrigated magnetic ablation catheter almost 10 years ago, catheter technology has significantly changed. New irrigation platforms, contact force sensing, and balloon-based technologies have entered the field, yet the magnetic catheter has remained unchanged in that time. There is ample opportunity to improve the magnetic catheter itself with increased magnetic force, improved handling, and irrigation, but this cannot occur without significant capital or a willing partner.

Despite these limitations, magnetic navigation seems to have found a niche in ventricular tachycardia and premature ventricular contraction (PVC) ablation. Magnetic navigation seems well suited to the ventricle. One of the limitations in the atrium is the relatively long stiff segment of the catheter; however, this is not a problem in the larger ventricle. One of the major advantages over manual navigation is the ability to access the entire ventricle with 
relative ease. Manual catheters are limited by fixed curves, which makes navigating portions of the left ventricle difficult, in some cases requiring deflectable sheaths or multi-curve catheters. This limitation also makes manual catheter contact force in certain areas inconsistent or suboptimal, which may also result in insufficient ablation lesions. Maps obtained via magnetic navigation tend to have less distortion and more accurately represent the chamber of interest $[5,8]$. The more uniform and constant contact may also improve the ablation lesions. Mapping with a magnetic catheter also appears to produce less ectopy, which is particularly important in PVC ablation when a catheter-induced PVC could cause the operator to ablate off target [9].

Epicardial mapping and ablation has been shown to be useful in some ventricular tachycardia such as arrhythmogenic right ventricular dysplasia (ARVD) [10]. Magnetic navigation has a particular advantage in this modality because altering the vectors, allows a more direct approach to mapping the epicardial surface. In addition, the vector can be directed toward the heart surface, which may reduce the collateral damage seen during epicardial ablation to adjacent structures. Similarly, in congenital heart disease, magnetic navigation may allow access to areas not reachable via manual navigation [11-14].

In the paper, "Comparison of Remote Magnetic Navigation Ablation and Manual Ablation of Idiopathic Ventricular Arrhythmia After Failed Manual Ablation," the authors studied a group of patients referred for ablation after having failed a previous ablation for ventricular arrhythmia. They found that the patients who underwent mapping and ablation using the remote magnetic navigation system had a significantly higher success rate (91 vs. $69 \% p=0.02$ ). This is a difficult population understanding that the patients were previously unsuccessfully ablated. In addition, as expected, fluoroscopy time was reduced. The authors also reported particular success in the posterior right ventricular outflow tract and posterior tricuspid valve area [15].

This is a retrospective analysis but does confirm that magnetic navigation appears to have a particular benefit in the ablation of ventricular arrhythmias. Despite the technological improvements in manual ablation catheters, magnetic navigation wins out in this study.

This win certainly enlightens us to the need for a prospective trial, understanding that magnetic navigation still lacks the most advanced catheter technology. If this is confirmed by such a trial, then it would suggest that with some technological advances, magnetic navigation could compete in other ablation realms. Certainly new catheter designs that improve magnetic force and handling would spur operators who use the system only for ventricular arrhythmias to expand to the left atrium if all the benefits were available in that chamber. If the system could be designed to allow for placement in existing laboratories at acceptable cost, then a vastly expanded group of potential operators would be created, perhaps incentivizing further investment into the technology and applications.

Remote magnetic navigation may be superior to manual navigation in ventricular arrhythmia ablation [7]. This needs to be verified by a multi-center randomized study. Currently, the MAGNETIC VT study (NCT02637947) is enrolling across the world to answer this question in ischemic cardiomyopathy patients with ventricular arrhythmias. More studies are needed to validate this hypothesis in other types of ventricular arrhythmias. Technological improvements are needed to keep magnetic navigation, clearly a useful tool for some, from unnecessarily dying out. The magnetic navigation machine may have won this round, but it is a long fight.

\section{References}

1. Akca F, Schwagten B, Theuns DAJ, Takens M, Musters P, SziliTorok T. Safety and feasibility of single-catheter ablation using remote magnetic navigation for treatment of slow-fast atrioventricular nodal reentrant tachycardia compared to conventional ablation strategies. Acta Cardiol. 2013;68(6):559-67.

2. Kim S-H, Oh Y-S, Kim D-H, Choi IJ, Kim T-S, Shin W-S, et al. Long-term outcomes of remote magnetic navigation for ablation of supraventricular tachycardias. J Interv Card Electrophysiol. 2015;43(2):187-92.

3. Wu J, Deisenhofer I, Ammar S, Fichtner S, Reents T, Zhu P, et al. Acute and long-term outcome after catheter ablation of supraventricular tachycardia in patients after the Mustard or Senning operation for D-transposition of the great arteries. Europace. 2013;15(6):886-91.

4. Jin QI, Pehrson S, Jacobsen PK, Chen XU. Efficacy and safety of atrial fibrillation ablation using remote magnetic navigation: experience from 1,006 procedures. J Cardiovasc Electrophysiol. 2016;27 Suppl 1:S23-28.

5. Weiss JP, May HT, Bair TL, Crandall BG, Cutler MJ, Day JD, et al. A comparison of remote magnetic irrigated tip ablation versus manual catheter irrigated tip catheter ablation with and without force sensing feedback. J Cardiovasc Electrophysiol. 2016;27 Suppl 1:S5-S10.

6. Jin Q, Pehrson S, Jacobsen PK, Chen X. Impact of catheter ablation with remote magnetic navigation on procedural outcomes in patients with persistent and long-standing persistent atrial fibrillation. J Interv Card Electrophysiol. 2015;44(2):197-204.

7. Zhang F, Yang B, Chen H, Ju W, Kojodjojo P, Cao K, et al. Magnetic versus manual catheter navigation for mapping and ablation of right ventricular outflow tract ventricular arrhythmias: a randomized controlled study. Heart Rhythm. 2013;10(8):1178-83.

8. Tahir SMA, Chaudhry GM, Syed MA, Marchese T, Kotler G, Haffajee CI, et al. Remote magnetic navigation system provides a superior catheter stability in acquisition of His bundle electrogram. J Interv Card Electrophysiol. 2008;21(3):209-13.

9. Aagaard P, Natale A, Briceno D, Nakagawa H, Mohanty S, Gianni $\mathrm{C}$, et al. Remote magnetic navigation: a focus on catheter ablation of ventricular arrhythmias. J Cardiovasc Electrophysiol. 2016;27 Suppl 1:S38-44.

10. Santangeli P, Dello Russo A, Pieroni M, Casella M, Di Biase L, Burkhardt JD, et al. Fragmented and delayed electrograms within fibrofatty scar predict arrhythmic events in arrhythmogenic right ventricular cardiomyopathy: results from a prospective risk stratification study. Heart Rhythm. 2012;9(8):1200-6. 
11. Di Biase L, Santangeli P, Astudillo V, Conti S, Mohanty P, Mohanty $\mathrm{S}$, et al. Endo-epicardial ablation of ventricular arrhythmias in the left ventricle with the remote magnetic navigation system and the 3.5-mm open irrigated magnetic catheter: results from a large single-center case-control series. Heart Rhythm. 2010;7(8):102935 .

12. Di Biase L, Santangeli P, Burkhardt DJ, Bai R, Mohanty P, Carbucicchio C, et al. Endo-epicardial homogenization of the scar versus limited substrate ablation for the treatment of electrical storms in patients with ischemic cardiomyopathy. J Am Coll Cardiol. 2012;60(2):132-41.
13. Roy K, Gomez-Pulido F, Ernst S. Remote magnetic navigation for catheter ablation in patients with congenital heart disease: a review. J Cardiovasc Electrophysiol. 2016;27 Suppl 1:S45-56.

14. Abraham P, Abkenari LD, Peters ECH, Szili-Torok T. Feasibility of remote magnetic navigation for epicardial ablation. Neth Heart J. 2013;21(9):391-5.

15. Kawamura M, Scheinman MM, Tseng ZH, Lee BK, Marcus GM, Badhwar N. Comparison of remote magnetic navigation ablation and manual ablation of idiopathic ventricular arrhythmia after failed manual ablation. J Interv Card Electrophysiol. 2016. doi:10.1007 /s10840-016-0158-x. 